P050

\section{MODELLO ANIMALE DI SINDROME DI GUILLAIN-BARRE' DA IMMUNIZZAZIONE CON LIPOPOLISACCARIDE DI CAMPYLOBACTER JEJUNI}

\author{
Gandolfi P.,* Luciani M.,* Caporale C.M.,** Armillotta G.,* \\ Uncini A.** \\ Istituto Zooprofilattico Sperimentale dell'Abruzzo e del Molise \\ "G.Caporale", Teramo * \\ Centro Malattie Neuromuscolari. Dipartimento di Oncologia e \\ Neuroscienze, Università "G. d'Annunzio", Chieti ***
}

La sindrome di Guillain-Barré (GBS) è una neuropatia disimmune post-infettiva. La gastroenterite da Campylobacter jejuni ( $C j$ ) è l'antecedente infettivo più comune nella variante assonale di GBS. I pazienti affetti da GBS assonale hanno frequentemente anticorpi contro gangliosidi ( GM1, GD1a ) espressi nel nervo periferico umano. Alcuni sierotipi di Campylobacter jejuni sintetizzano acido sialico e contengono epitopi gangliosidici nel lipopolisaccaride (LPS). E' stato ipotizzato che gli anticorpi antiganglioside indotti dall'infezione da $C j$ aggredirebbero i gangliosidi del nervo provocando la GBS. Abbiamo recentemente sviluppato nel coniglio un modello di GBS con elevati titoli di anticorpi antiganglioside tramite immunizzazione con lipopolisaccaride estratto da $C j$ e keyhole limpet hemocyanin (KLH). Il modello animale ripercorre il percorso patogenetico ipotizzato nella GBS post-infettiva umana e la KLH, una glicoproteina che stimola in maniera non specifica l'immunità umorale $\mathrm{e}$ cellulare, svolge un ruolo cruciale nell'induzione della malattia.

Abbiamo immunizzato undici conigli con LPS, estratto da un ceppo di Campylobacter jejuni HS:19, e adiuvante di Freund (CFA) (I gruppo); in un secondo esperimento abbiamo immunizzato sette conigli con LPS, CFA e KLH

(II gruppo). Tutti i conigli del primo e del secondo gruppo hanno dato luogo ad un'importante risposta umorale nei confronti del LPS. Nel gruppo I le IgG hanno raggiunto un titolo di 25600 e di 6400 nel gruppo II. Anticorpi IgM anti-GM1 sono stati trovati a basso titolo due settimane dopo la prima immunizzazione in entrambi i gruppi e in seguito sono aumentati fino a 3200 nel primo gruppo e a 6400 nel secondo. Le IgG anti- GM1 allo stesso modo sono aumentate fino a 51200 nel gruppo I e a 25600 nel gruppo II. Attraverso la caratterizzazione del LPS mediante Western Immunoblotting si è visto che il siero dei conigli immunizzati reagisce fortemente con una banda di migrazione di $6.5 \mathrm{kDa}$ così come con la stessa banda reagiscono la tossina colerica (CT), la peanut arabic agglutinin (PNA) e il siero del paziente con anticorpi anti-GM1. Allo stesso modo abbiamo dimostrato in Western Immunoblotting il legame tra il LPS e gli anticorpi monoclonali anti-GM1, GD1a e GD1b. La cinetica di IgM e IgG anti-GDlb si è mostrata simile a quella degli anticorpi antiGM1 ma i titoli sono stati più bassi: le IgG sono aumentate fino a 3200 nel primo gruppo e fino a 12800 nel secondo. Le IgM anti-GDla sono rimaste a basso titolo in entrambi i gruppi dall'inizio alla fine dell'esperimento mentre le IgG anti-GDla sono aumentate fino a 3200 nel primo gruppo e a 800 nel secondo. Non abbiamo trovato invece in nessuno dei due gruppi IgM e IgG anti-GD1b.

Questo è stato il primo modello animale che riproduce il processo patogenetico ipotizzato in GBS di tipo assonale con produzione di anticorpi antiganglioside in seguito ad un'infezione da $C$.jejuni; si è visto che il LPS di $C j$ è un potente stimolatore capace di indurre nel coniglio un'importante risposta antiganglioside. Inoltre si è anche visto che, per indurre la neuropatia, è di importanza cruciale l'impiego di $\mathrm{KLH}$, una glicoproteina conosciuta come stimolatrice di risposta sia umorale che cellulare.

\section{P051}

\section{TREND DELLA SENSIBILITÀ DI BATTERI NON FERMENTANTI ISOLATI DALL'ESPETTORATO DI PAZIENTI CON FIBROSI CISTICA (200I-2003)}

Garlaschi M.L.*, Cariani L.*, Scarazatti M.^, Clarizia G.*, Russo P.*, Laricchia L.*, Costantini D.**.

* U.O. di Microbiologia, Ist. Clin. di Perfezion., Milano

** Dip. di Pediatria, Centro di Riferimento Regionale

Fibrosi Cistica, Cl. Ped "De Marchi", Univ. Milano

^ Dip. di Pediatria, Cl. Ped "De Marchi", Univ. Milano

Introduzione. I microrganismi che colonizzano le basse vie aeree di pazienti con Fibrosi Cistica nei primi anni di vita, sono Haemophilus influenzae e Streptococcus pneumoniae. Nell'età prepubere si instaura Staphylococcus aureus e poi, nell'età adolescenziale si instaurano i batteri Gram negativi non fermentanti, fra questi Pseudomonas aeruginosa (PA), che segna un netto declino della funzionalità respiratoria, Burkholderia cepacia complex (BC), che spesso determina una prognosi sfavorevole, Acrhomobacter xylosoxidans (AC) e Stenotrophomonas maltophilia (SM) di non ancora chiaro ruolo patogeno.

Materiali e metodi. Sono stati valutati 650 pazienti con Fibrosi Cistica afferenti al Centro di Riferimento Regionale Lombardo. Per ogni paziente è stato eseguito l'esame colturale dell'espettorato almeno due volte in un anno, per un totale di 7959 campioni, di cui $1440(82 \%)$ è risultato positivo per uno o più microrganismi.

I batteri non fermentanti valutati nei tre anni sono stati 12.738 .

Risultati. Nella tabella sottostante sono messe a confronto le $\%$ di sensibilità, dei batteri Gram negativi non fermentanti sopra riportati, negli anni 2001-2003 verso gli antibiotici più utilizzati e più significativi:

\begin{tabular}{lcccccccc}
\hline & \multicolumn{2}{c}{ PA } & \multicolumn{2}{c}{ BC } & \multicolumn{2}{c}{ AX } & \multicolumn{2}{c}{ SM } \\
\hline & ${ }^{\prime} 01$ & ${ }^{\prime} 03$ & ${ }^{\prime} 01$ & ${ }^{2} 03$ & ${ }^{\prime} 01$ & ${ }^{\prime} 03$ & ${ }^{\prime} 01$ & ${ }^{\prime} 03$ \\
\hline Amikacina & 55 & 52 & 0 & 0 & 4 & 6 & $\mathrm{NV}^{*}$ & $\mathrm{Nv}$ \\
\hline Ceftazidime & 65 & 60 & 3 & 8 & 40 & 40 & $\mathrm{Nv}$ & $\mathrm{Nv}$ \\
\hline Cprofloxac. & 54 & 57 & 8 & 1 & 5 & 4 & $\mathrm{Nv}$ & $\mathrm{Nv}$ \\
\hline Levofloxac. & 54 & 57 & 15 & 5 & 31 & 32 & $\mathrm{Nv}$ & $\mathrm{Nv}$ \\
\hline Meropenem & 80 & 76 & 37 & 40 & 57 & 67 & $\mathrm{Nv}$ & $\mathrm{Nv}$ \\
\hline Pip/Tazob. & 61 & 81 & 15 & 9 & 66 & 53 & $\mathrm{Nv}$ & $\mathrm{Nv}$ \\
\hline Tobramicina & 65 & 64 & 0 & 0 & 3 & 2 & $\mathrm{Nv}$ & $\mathrm{Nv}$ \\
\hline Trim./Sulfa & 0 & 0 & 36 & 28 & 58 & 75 & 93 & 95 \\
\hline
\end{tabular}

$* \mathrm{Nv}=$ non valutato

\section{Conclusioni.}

Nessuno dei microrganismi considerati, ha mostrato un incremento di resistenza verso gli antibiotici valutati, negli anni 2001, 2003.

Bibliografia.

1. Cystic Fibrosis Foundation. 2002. Patient registry 2001 annual report. Cystic Fibrosis Foundation, Washington, D.C 